\title{
Morbidity of central neck dissection for papillary thyroid cancer
}

\author{
Davide Lombardi $^{1}$, Remo Accorona ${ }^{1}$, Alberto Paderno ${ }^{1}$, Carlo Cappelli ${ }^{2}$, Piero Nicolai ${ }^{1}$ \\ ${ }^{1}$ Department of Otorhinolaryngology - Head and Neck Surgery, ${ }^{2}$ Department of Medical and Surgical Sciences, Endocrine and Metabolic Unit, \\ University of Brescia, Brescia, Italy \\ Contributions: (I) Conception and design: D Lombardi, R Accorona; (II) Administrative support: P Nicolai; (III) Provision of study materials or \\ patients: D Lombardi, R Accorona, A Paderno; (IV) Collection and assembly of data: D Lombardi; R Accorona; A Paderno; (V) Data analysis and \\ interpretation: D Lombardi; R Accorona; C Cappelli; (VI) Manuscript writing: All authors; (VII) Final approval of manuscript: All authors. \\ Correspondence to: Davide Lombardi, MD. Department of Otorhinolaryngology - Head and Neck Surgery, University of Brescia, Piazza Spedali Civili \\ 1, 25100 Brescia, Italy. Email: davinter@libero.it.
}

\begin{abstract}
Thyroid cancer has a very well-known propensity for nodal involvement, either in the central and lateral neck compartments. Neck dissection addressing the central compartment may be performed with an elective or therapeutic intent, the former concomitantly to a thyroidectomy whereas the latter may be accomplished also as a revision procedure for recurrent disease. In this paper complications of central compartment neck dissection will be described, analyzing separately primary and revision procedures.
\end{abstract}

Keywords: Central compartment; neck dissection; thyroid cancer

Submitted Apr 19, 2017. Accepted for publication Apr 27, 2017.

doi: $10.21037 /$ gs.2017.05.07

View this article at: http://dx.doi.org/10.21037/gs.2017.05.07

\section{Introduction}

Papillary thyroid cancer (PTC) has the peculiar tendency to spread to central and lateral neck lymph nodes. PTC recurrences typically involve cervical lymph nodes in $60-75 \%$ of cases, with the central compartment being the most frequently involved site (1-3). While no controversy exists on the role of therapeutic central lymph node dissection (CND), concomitant with thyroidectomy or as a revision procedure, elective CND is still a debated issue. Indeed, the American Thyroid Association (ATA) guidelines (4) do not help in the controversy on elective treatment of level VI, due to an unclear prognostic role and a non-negligible rate of complications. The debate on morbidity of CND is prevalently focused on injury, transient or permanent, to the recurrent laryngeal nerve (RLN) and/or parathyroid glands. Chyle leakage is rare, and data in the literature are consequently scarce. No specific data have been reported on other complications such as hematoma, vascular or visceral injury, and wound infection.

There are important differences in the surgical technique and complication rate between CND associated with total thyroidectomy (TTCND) and revision CND (RCND); for this reason, we will provide a separate discussion for the two topics.

\section{Surgical boundaries of the central neck compartment}

The ATA consensus statement regarding the terminology and classification of the central neck $(5,6)$ states that the CND contains the perithyroidal and paratracheal soft tissues as well as lymph nodes; it is limited superiorly by the hyoid bone, laterally by the common carotid arteries, anteriorly the by the superficial layer of the deep cervical fascia (and posterior aspect of the infrahyoid muscles), posteriorly by the deep layer of the cervical fascia (covering the prevertebral muscles and the oesophagus), and inferiorly by sternum and the innominate artery. The term CND, as defined by the ATA consensus, implies removal of prelaryngeal and pretracheal lymphatic tissue and at least one paratracheal lymph node. This is defined as unilateral CND, whereas in bilateral CND paratracheal nodes on both sides are removed $(5,6)$. 


\section{Complications in CND associated with thyroidectomy}

In recent years, the "pros" and "cons" of elective central compartment neck dissection were thoroughly analyzed but a significant disputation still remains: several authors, indeed, have suggested routine use of elective CND in differentiated thyroid carcinomas (7-10). On the other hand, despite the high prevalence of lymph node micrometastases from PTC (ranging from $38 \%$ to $61 \%$ ) $(1,3,11$ ), several investigators (11-15) have outlined the limits of such an approach, with a significant increase in the morbidity associated with standard total thyroidectomy and no differences in overall and disease specific survival.

One of the main reasons that led several authors to consider elective CND is the low accuracy of preoperative diagnostic tools in identifying nodal involvement at this level, especially when dealing with recurrent disease (16). Indeed, while neck ultrasonography (US) and cross-sectional imaging techniques, such as computed tomography (CT) and magnetic resonance imaging (MRI), reliably evaluate lateral neck levels, they are less accurate in the assessment of the central compartment. Several studies have reported a low sensitivity of US and CT, with values ranging from $51 \%$ to $67 \%$, and from $62 \%$ to $66 \%$, respectively $(17,18)$; this is due to the fact that lymph nodes are often small and located deeply in the neck, or just posterior to the sternum, thus hindering adequate visualization $(13,19-21)$.

In 2006, the European Consensus Statement (22) emphasized that the benefits of elective CND in the absence of pre- or intraoperative evidence for nodal disease are controversial: in fact, there is no evidence that it decreases the rate of either recurrence or mortality; conversely, it may allow accurate staging of the disease that can modulate subsequent treatments and follow-up. Similarly, recommendations in the 2015 American Guidelines (4) are not clear-cut. Recommendation \#36 suggests elective CND (ipsilateral or bilateral) in patients with PTC with clinically uninvolved central neck lymph nodes (cN0) and advanced primary tumors (T3 or T4) or clinically involved lateral neck nodes (cN1b), or if the information might be used to plan further steps in therapy (4).

Most authors agree with the observation that TTCND leads to a higher risk of complications when compared with total thyroidectomy alone, particularly with regards to hypoparathyroidism (HPT) rather than RLN injury (11,12,17,23-29). In fact, the RLN at the end of thyroidectomy is already exposed, visible, and only its most caudal part needs to be further dissected $(17,25-27,29,30)$. For this reason, intraoperative nerve monitoring (IONM), despite its importance in the thyroidectomy phase, does not seem to play such an important role in RLN preservation during the CND phase (31). However, it is worth remembering that IONM may be helpful in identifying a loss of signal on the first side that could suggest to stage completion thyroidectomy, especially in case of nonaggressive thyroid cancer.

In the literature, the rate of transient RLN injury and HPT ranges from $0 \%$ to $7.3 \%$, and from $8.7 \%$ to $86 \%$, respectively $(11,12,15,23,30,32)$. Furthermore, the reported rates of permanent unintentional RLN injury and permanent HPT ranges from $0 \%$ to $5.9 \%$, and from $0 \%$ to $16.2 \%$, respectively (Table 1 ). Different authors suggested that the rate of complications in patients treated with TTCND may be overestimated $(15,32,47,48)$. In a pivotal paper by Giordano and colleagues (7), a comparative study on 1,087 patients affected by PTC with clinically negative lymph nodes was performed. Patients were divided in three homogeneous groups: patients treated with total thyroidectomy alone, unilateral TTCND, and bilateral TTCND. Overall, the results of the three groups were comparable to those reported in the literature: indeed, rates of transient RLN injury and HPT were $4.2 \%$ and $37.5 \%$, respectively. Regarding permanent complications, the rates of RLN injury and HPT were $1.2 \%$ and $9.4 \%$, respectively. The analysis comparing the three groups showed that neither unilateral nor bilateral TTCND were associated with a significantly increased rate of transient or permanent RLN injury. On the other hand, the rate of transient HPT was significantly higher in both unilateral and bilateral TTCND. Finally, the rate of permanent HPT was significantly increased in case of bilateral, but not unilateral, TTCND. These results were consistent with those reported in other studies $(11,12,17,23-29)$. The increased rate of permanent HPT in bilateral TTCND may be due to the increased risk of traumatic and ischemic damage to parathyroid glands caused by surgical manipulation $(7,30,49)$; therefore, it is strongly suggested to make every effort to preserve the vascular supply to the superior parathyroid gland. On the contrary, it may be more technically demanding to avoid ischemic injuries to the inferior parathyroid gland $(1,7,11,25)$. It is worth mentioning that several authors demonstrated no significant effect on postoperative HPT in case of intraoperative ligation of the inferior thyroid artery (ITA) (50-53), although the preservation of the inferior thyroidal 
Table 1 Review of the literature showing the rate of surgical complications after TTCND

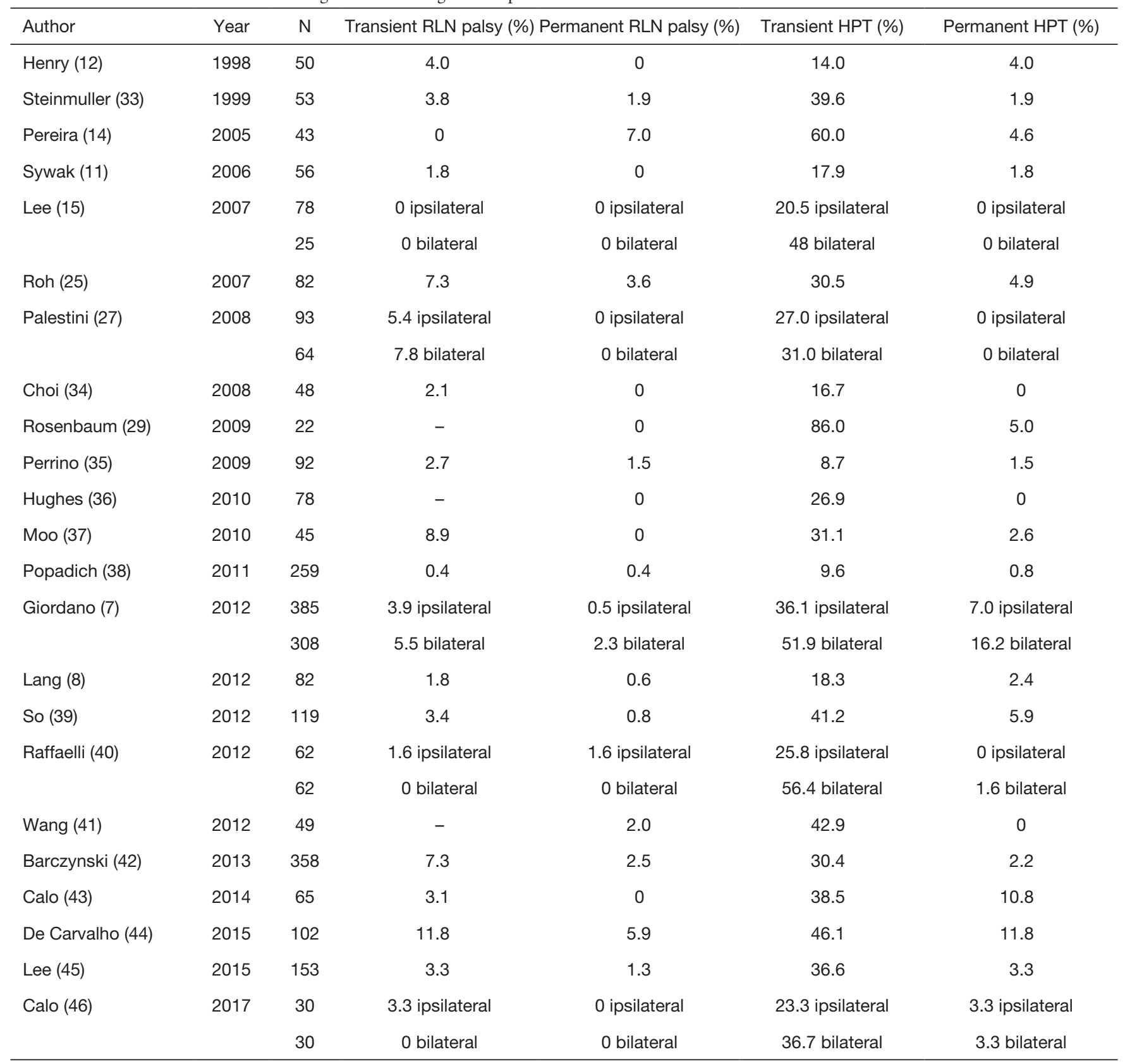

vein (ITV) seems to reduce postoperative hypocalcemia (54). In two papers, this finding was ascribed to a damage caused by insufficient blood discharge to whom a parathyroid is exposed if the ITV is sacrificed far from the thyroid parenchyma during CND $(54,55)$.

Nevertheless, the inferior parathyroid gland can be removed and, after intraoperative histologic confirmation by frozen sections, re-implanted as an autograft in the sternal belly of the sternocleidomastoid muscle. Indeed, both Smith et al. (56) and Shaha et al. (57) showed conclusive data on the efficacy of parathyroid autotransplantation to reduce the risk of postoperative HPT when aggressive dissection, or vascular injury to the parathyroids, is performed.

Since the risk of central compartment involvement is mainly on the same side of the tumor (58) and contralateral metastases, conversely, are rare without ipsilateral positive 
nodes, several authors have recommended to perform TTCND ipsilateral to the tumor in case of differentiated thyroid carcinomas, thus finding a reasonable balance between morbidity and oncologic outcome (7-10).

The results presented by Giordano and colleagues (7), were also confirmed in a recent meta-analysis published by Zhao et al. (59). Patients who underwent TTCND with elective intent had a significantly increased risk of postoperative hypocalcemia than patients who underwent total thyroidectomy alone, including temporary hypocalcemia $(28.7 \%$ vs. $17.5 \%$, OR $=2.37)$, and permanent hypocalcemia $(4.1 \%$ vs. $2.3 \%, \mathrm{OR}=1.93)$. On the other hand, the risk of temporary and permanent RLN injury was confirmed to be similar in the two groups $(\mathrm{OR}=1.22$ and 1.17 respectively) (59). However, regarding overall complications, the meta-analysis indicated that the TTCND group has a significantly increased overall morbidity $(\mathrm{OR}=2.56)$, although it was similar after excluding temporary hypocalcemia $(\mathrm{OR}=1.14)$.

Chyle leakage is a rare complication of thyroid surgery, with or without $\mathrm{CND}$, with an estimated prevalence of less than 1\% (60-63). Lee et al. (61) retrospectively analyzed 2,314 patients who underwent TTCND (unilateral or bilateral) for thyroid carcinoma: $14(0.6 \%)$ patients were reported to have postoperative chyle leak, with a mean duration of the leakage of 4.3 days and a mean chyle volume of $53.2 \mathrm{~mL} /$ day. Before this paper, to the best of our knowledge, only a single case had been reported in the literature (60). All patients were treated conservatively by pressure dressings, a fat-free diet, and repeated percutaneous drainage. The authors ascribed the chyle leakage in patients who did not undergo lateral neck dissection to the presence of minor lymphatic ducts in the central compartment. These small ducts, when injured during dissection, may immediately collapse, and thus chyle leakage may be not intraoperatively detected. Due to its limited entity, chyle leak is commonly managed with conservative maneuvers and revision surgery is generally not required.

\section{Complications in revision CND}

Recurrent disease in the neck central compartment should be suspected in presence of lesions greater than $8 \mathrm{~mm}$ at US; these lesions should be considered for cytologic evaluation, Thyroglobulin aspirate rinsing analysis, and eventually, revision surgery (16). Surgery for recurrent disease in the central neck compartment is a complex procedure, and specific data regarding surgical morbidity are scarce, mainly as a result of the limited number of patients requiring $\mathrm{RCND}(64,65)$.

Indications for revision surgery in central neck compartment include recurrent metastatic thyroid carcinoma to central compartment lymph nodes and inadequate initial surgery $(5,66)$. RCND is technically demanding because of the abundant scar tissue and distorted anatomy, which may lead to a higher risk of injury to the RLN and parathyroid glands. RLN may be, indeed, in a less predictable location after thyroidectomy; in addition, the parathyroid glands and RLN may be encased within fibrotic tissue, making them indistinguishable from the tumor (16).

Central neck lymph node metastases are usually detected during follow-up by means of increased serum Thyroglobulin levels and by radiological examinations (US, radioactive iodine scan, or PET-CT), and subsequently confirmed with fine-needle aspiration cytology (FNAC) and contrast enhanced MRI or CT $(18,21,67)$.

Several series have reported a higher incidence of permanent RLN palsy in RCND compared to primary setting procedures, with rates ranging from $1 \%$ to $12 \%$ $(64,65,68-70)$. Regarding HPT, in RCND the parathyroid glands are at increased risk for devascularization or inadvertent removal. Several series $(1,68,71-73)$ have reported an incidence of temporary and permanent HPT after revision surgery, ranging from $0.3 \%$ to $15 \%$, and from $0 \%$ to $4.9 \%$, respectively. Kim et al. (64) reported their experience of RCND on 20 patients. All patients had a normally preoperatively and postoperatively functioning larynx. Furthermore, 4 (20\%) patients experienced transient postoperative HPT, with no evidence of permanent HPT. The authors emphasised the importance of a meticulous surgical dissection with identification of RLN in the lower part of tracheoesophageal groove, and the use of IONM. More recently, Cayonu et al. (65) retrospectively revised a single center series of 61 patients treated for central compartment lymph node recurrence from thyroid cancer. The authors suggested a step-by-step technique for identification and preservation of RLN and parathyroid glands. Interestingly, they did not routinely use IONM. They reported an acceptable rate of complications: temporary RLN palsy in 4 patients $(6.6 \%)$, temporary HPT in 8 (13.1\%), and permanent HPT in $3(4.9 \%)$. No permanent RLN palsy, wound infection, or hematoma were reported.

Onkendi et al. (74) reported their experience in 410 patients operated for recurrent PTC. One-hundred-twelve patients underwent unilateral and 107 bilateral CND (58\% 
of the series) with $80 \%$ of positive lymph nodes at definitive histologic examination. Unfortunately, an important limitation of the study is that the rate of complications is calculated on the whole series, including revision surgery on thyroid gland and lateral neck dissection: the overall complications rate was $6 \%$, with $3 \%$ permanent HPT, and $0.5 \%$ permanent RLN.

In general, IONM can be extremely useful because it facilitates the identification and dissection of the nerve in a surgical field with distorted landmarks, and may be helpful in predicting nerve function after surgery (16). Indeed, it is possible to map, with IONM's probe, the area where the nerve might be located to avoid blind dissection. However, according to the results of a recent comparative analysis, the use of IONM in thyroid revision surgery does not seem to significantly reduce the risk of RLN palsy (31); careful dissection and visualization of the nerve remain of pivotal importance to minimize the risk of iatrogenic injury. The authors also stated that, in RCND, most problems are related to the difficult dissection of noble structures from scar tissue. In this sense, Alesina et al. (31) suggested that continuous monitoring could be very useful in minimizing the risk of injury.

Although in primary surgery preservation of the ITA does not seem, as already mentioned, to be great help in preventing postoperative HPT, Kim et al. (64) recommended preserving the artery in RCND to minimize the risk of devascularization of the parathyroid glands. However, great attention is commonly placed on the outcomes and usefulness of parathyroid autotransplantation. Indeed, to avoid postoperative HPT, several authors have recommended to carefully examine the specimen for parathyroid tissue by means of a histologic frozen section analysis $(64,65,74)$. Reimplantation of the parathyroid gland(s) into the sternocleidomastoid muscle at the time of revision CND will decrease the risk of long-term permanent HPT (64). It is worth remembering that fibrosis and multiple positive lymph nodes in the dissected specimen can make identification and confirmation of parathyroid tissue very difficult. In patients with extensive extracapsular lymph node spread and multiple involved nodes, reimplantation can inadvertently lead to reimplantation of tumor along with parathyroid tissue (64). Notably, also in recurrent disease, in absence of bilateral central compartment involvement, it is preferable to avoid bilateral CND in order to minimize the risk of definitive HPT and bilateral RLN palsy (16).

As a last remark, the most important element in minimizing complications in thyroid surgery is the surgical volume of the surgeon, as widely reported in the literature $(28,75,76)$. For this reason, Mazzaferri and colleagues (28) have properly stated that it is overall better, for the small minority of patients who subsequently develop central node metastasis to undergo therapeutic RCND by more experienced thyroid surgeons, than all patients undergo prophylactic dissection, with the majority being performed by less experienced surgeons.

\section{Conclusions}

Primary CND is associated with an acceptable rate of complications, since an increase of permanent HPT is reported when CND is performed bilaterally. Preservation of the vascular pedicle of the parathyroid glands, in particular the ITV, prevents ischemic damage. RCND for recurrent or persistent thyroid cancer is a challenging procedure even for the most experienced surgeon. Surgical dissection must be meticulous, with identification of the RLN and parathyroid glands. IONM may help in identifying the nerve in a fibrotic surgical field, thus minimizing the risk of iatrogenic damage. In both TTCND and RCND, parathyroid autotransplantation seems to contribute to prevention of postoperative HPT. Other complications, such as chyle leakage, are very rare and data in the current literature are scarce. Surgical volume remains the most important factor affecting the rate of complications in thyroidectomy and/or CND.

\section{Acknowledgements}

None.

\section{Footnote}

Conflicts of Interest: The authors have no conflicts of interest to declare.

\section{References}

1. Tufano RP, Bishop J, Wu G. Reoperative central compartment dissection for patients with recurrent/ persistent papillary thyroid cancer: efficacy, safety, and the association of the BRAF mutation. Laryngoscope 2012;122:1634-40.

2. Goyal N, Pakdaman M, Kamani D, et al. Mapping the distribution of nodal metastases in papillary thyroid 
carcinoma: Where exactly are the nodes? Laryngoscope 2017;127:1959-64.

3. Mazzaferri EL, Robyn J. Postsurgical management of differentiated thyroid carcinoma. Otolaryngol Clin North Am 1996;29:637-62.

4. Haugen BR, Alexander EK, Bible KC, et al. 2015 American Thyroid Association Management Guidelines for Adult Patients with Thyroid Nodules and Differentiated Thyroid Cancer: The American Thyroid Association Guidelines Task Force on Thyroid Nodules and Differentiated Thyroid Cancer. Thyroid 2016;26:1-133.

5. American Thyroid Association Surgery Working Group, American Association of Endocrine Surgeons, American Academy of Otolaryngology-Head and Neck Surgery, et al. Consensus statement on the terminology and classification of central neck dissection for thyroid cancer. Thyroid 2009;19:1153-8.

6. Orloff LA, Kuppersmith RB. American Thyroid Association's central neck dissection terminology and classification for thyroid cancer consensus statement. Otolaryngol Head Neck Surg 2010;142:4-5.

7. Giordano D, Valcavi R, Thompson GB, et al. Complications of central neck dissection in patients with papillary thyroid carcinoma: results of a study on 1087 patients and review of the literature. Thyroid 2012;22:911-7.

8. Lang BH, Wong KP, Wan KY, et al. Impact of routine unilateral central neck dissection on preablative and postablative stimulated thyroglobulin levels after total thyroidectomy in papillary thyroid carcinoma. Ann Surg Oncol 2012;19:60-7.

9. Chan AC, Lang BH, Wong KP. The pros and cons of routine central compartment neck dissection for clinically nodal negative (cN0) papillary thyroid cancer. Gland Surg 2013;2:186-95.

10. Hartl DM, Mamelle E, Borget I, et al. Influence of prophylactic neck dissection on rate of retreatment for papillary thyroid carcinoma. World J Surg 2013;37:1951-8.

11. Sywak M, Cornford L, Roach P, et al. Routine ipsilateral level VI lymphadenectomy reduces postoperative thyroglobulin levels in papillary thyroid cancer. Surgery 2006;140:1000-5; discussion 1005-7.

12. Henry JF, Gramatica L, Denizot A, et al. Morbidity of prophylactic lymph node dissection in the central neck area in patients with papillary thyroid carcinoma. Langenbecks Arch Surg 1998;383:167-169.

13. Wada N, Duh QY, Sugino K, et al. Lymph node metastasis from 259 papillary thyroid microcarcinomas: frequency, pattern of occurrence and recurrence, and optimal strategy for neck dissection. Ann Surg 2003;237:399-407.

14. Pereira JA, Jimeno J, Miquel J, et al. Nodal yield, morbidity, and recurrence after central neck dissection for papillary thyroid carcinoma. Surgery 2005;138:1095-100, discussion 1100-1.

15. Lee YS, Kim SW, Kim SW, et al. Extent of routine central lymph node dissection with small papillary thyroid carcinoma. World J Surg 2007;31:1954-9.

16. Scharpf J, Tuttle M, Wong R, et al. Comprehensive management of recurrent thyroid cancer: An American Head and Neck Society consensus statement: AHNS consensus statement. Head Neck 2016;38:1862-9.

17. Roh JL, Park JY, Park CI. Prevention of postoperative hypocalcemia with routine oral calcium and vitamin D supplements in patients with differentiated papillary thyroid carcinoma undergoing total thyroidectomy plus central neck dissection. Cancer 2009;115:251-8.

18. Kim E, Park JS, Son KR, et al. Preoperative diagnosis of cervical metastatic lymph nodes in papillary thyroid carcinoma: comparison of ultrasound, computed tomography, and combined ultrasound with computed tomography. Thyroid 2008;18:411-8.

19. Leboulleux S, Girard E, Rose M, et al. Ultrasound criteria of malignancy for cervical lymph nodes in patients followed up for differentiated thyroid cancer. J Clin Endocrinol Metab 2007;92:3590-4.

20. Marshall CL, Lee JE, Xing Y, et al. Routine pre-operative ultrasonography for papillary thyroid cancer: effects on cervical recurrence. Surgery 2009;146:1063-72.

21. Hwang HS, Orloff LA. Efficacy of preoperative neck ultrasound in the detection of cervical lymph node metastasis from thyroid cancer. Laryngoscope 2011;121:487-91.

22. Pacini F, Schlumberger M, Dralle H, et al. European consensus for the management of patients with differentiated thyroid carcinoma of the follicular epithelium. Eur J Endocrinol 2006;154:787-803.

23. Cheah WK, Arici C, Ituarte PH, et al. Complications of neck dissection for thyroid cancer. World J Surg 2002;26:1013-6.

24. Cavicchi O, Piccin O, Caliceti U, et al. Transient hypoparathyroidism following thyroidectomy: a prospective study and multivariate analysis of 604 consecutive patients. Otolaryngol Head Neck Surg 2007;137:654-8.

25. Roh JL, Park JY, Park CI. Total thyroidectomy plus neck dissection in differentiated papillary thyroid carcinoma patients: pattern of nodal metastasis, morbidity, recurrence, 
and postoperative levels of serum parathyroid hormone. Ann Surg 2007;245:604-610.

26. Bardet S, Malville E, Rame JP, et al. Macroscopic lymphnode involvement and neck dissection predict lymphnode recurrence in papillary thyroid carcinoma. Eur J Endocrinol 2008;158:551-60.

27. Palestini N, Borasi A, Cestino L, et al. Is central neck dissection a safe procedure in the treatment of papillary thyroid cancer? Our experience. Langenbecks Arch Surg 2008;393:693-8.

28. Mazzaferri EL, Doherty GM, Steward DL. The pros and cons of prophylactic central compartment lymph node dissection for papillary thyroid carcinoma. Thyroid 2009;19:683-9.

29. Rosenbaum MA, McHenry CR. Central neck dissection for papillary thyroid cancer. Arch Otolaryngol Head Neck Surg 2009;135:1092-7.

30. Ardito G, Revelli L, Polistena A, et al. Complications of Neck Dissections in Papillary Thyroid Carcinoma: A Modified Procedure to Reduce Parathyroid Morbidity. In Vivo 2016;30:303-8.

31. Alesina PF, Rolfs T, Hommeltenberg S, et al. Intraoperative neuromonitoring does not reduce the incidence of recurrent laryngeal nerve palsy in thyroid reoperations: results of a retrospective comparative analysis. World J Surg 2012;36:1348-53.

32. Goropoulos A, Karamoshos K, Christodoulou A, et al. Value of the cervical compartments in the surgical treatment of papillary thyroid carcinoma. World J Surg 2004;28:1275-1281.

33. Steinmüller T, Klupp J, Wenking S, et al. Complications associated with different surgical approaches to differentiated thyroid carcinoma. Langenbecks Arch Surg 1999;384:50-3.

34. Choi SJ, Kim TY, Lee JC, et al. Is routine central neck dissection necessary for the treatment of papillary thyroid microcarcinoma? Clin Exp Otorhinolaryngol 2008;1:41-5.

35. Perrino M, Vannucchi G, Vicentini L, et al. Outcome predictors and impact of central node dissection and radiometabolic treatments in papillary thyroid cancers $<$ or $=2 \mathrm{~cm}$. Endocr Relat Cancer 2009;16:201-10.

36. Hughes DT, White ML, Miller BS, et al. Influence of prophylactic central lymph node dissection on postoperative thyroglobulin levels and radioiodine treatment in papillary thyroid cancer. Surgery 2010;148:1100-6; discussion 1006-107.

37. Moo TA, McGill J, Allendorf J, et al. Impact of prophylactic central neck lymph node dissection on early recurrence in papillary thyroid carcinoma. World J Surg 2010;34:1187-91.

38. Popadich A, Levin O, Lee JC, et al. A multicenter cohort study of total thyroidectomy and routine central lymph node dissection for $\mathrm{cN} 0$ papillary thyroid cancer. Surgery 2011;150:1048-57.

39. So YK, Seo MY, Son YI. Prophylactic central lymph node dissection for clinically node-negative papillary thyroid microcarcinoma: influence on serum thyroglobulin level, recurrence rate, and postoperative complications. Surgery 2012;151:192-8.

40. Raffaelli M, De Crea C, Sessa L, et al. Prospective evaluation of total thyroidectomy versus ipsilateral versus bilateral central neck dissection in patients with clinically node-negative papillary thyroid carcinoma. Surgery 2012;152:957-64.

41. Wang TS, Evans DB, Fareau GG, et al. Effect of prophylactic central compartment neck dissection on serum thyroglobulin and recommendations for adjuvant radioactive iodine in patients with differentiated thyroid cancer. Ann Surg Oncol 2012;19:4217-22.

42. Barczyński M, Konturek A, Stopa M, et al. Prophylactic central neck dissection for papillary thyroid cancer. Br J Surg 2013;100:410-8.

43. Calò PG, Pisano G, Medas F, et al. Total thyroidectomy without prophylactic central neck dissection in clinically node-negative papillary thyroid cancer: is it an adequate treatment? World J Surg Oncol 2014;12:152.

44. Ywata de Carvalho A, Chulam TC, Kowalski LP. Longterm Results of Observation vs Prophylactic Selective Level VI Neck Dissection for Papillary Thyroid Carcinoma at a Cancer Center. JAMA Otolaryngol Head Neck Surg 2015; 141:599-606.

45. Lee DY, Oh KH, Cho JG, et al. The Benefits and Risks of Prophylactic Central Neck Dissection for Papillary Thyroid Carcinoma: Prospective Cohort Study. Int J Endocrinol 2015;2015:571480.

46. Calò PG, Conzo G, Raffaelli M, et al. Total thyroidectomy alone versus ipsilateral versus bilateral prophylactic central neck dissection in clinically node-negative differentiated thyroid carcinoma. A retrospective multicenter study. Eur J Surg Oncol 2017;43:126-32.

47. Asari R, Passler C, Kaczirek K, et al. Hypoparathyroidism after total thyroidectomy: a prospective study. Arch Surg 2008;143:132-7; discussion 138.

48. Chisholm EJ, Kulinskaya E, Tolley NS. Systematic review 
and meta-analysis of the adverse effects of thyroidectomy combined with central neck dissection as compared with thyroidectomy alone. Laryngoscope 2009;119:1135-9.

49. Promberger R, Ott J, Kober F, et al. Normal parathyroid hormone levels do not exclude permanent hypoparathyroidism after thyroidectomy. Thyroid 2011;21:145-50

50. Araujo Filho VJ, Silva Filho GB, Brandão LG, et al. The importance of the ligation of the inferior thyroid artery in parathyroid function after subtotal thyroidectomy. Rev Hosp Clin Fac Med Sao Paulo 2000;55:113-20.

51. Dolapçi M, Doganay M, Reis E, et al. Truncal ligation of the inferior thyroid arteries does not affect the incidence of hypocalcaemia after thyroidectomy. Eur J Surg 2000;166:286-8.

52. Maralcan G, Sayin Z, Baskonu I, et al. Does truncal ligation of the inferior thyroid arteries during bilateral subtotal thyroidectomy affect serum calcium levels? A prospective, randomized, controlled study. Int Surg 2006;91:211-6.

53. Chaudhary IA, Afridi Zu, Samiullah, et al. To ligate or not the inferior thyroid artery to avoid hypocalcaemia after thyroid surgery. J Ayub Med Coll Abbottabad 2007;19:19-22.

54. Lee DY, Cha W, Jeong WJ, et al. Preservation of the inferior thyroidal vein reduces post-thyroidectomy hypocalcemia. Laryngoscope 2014;124:1272-7.

55. Cocchiara G, Cajozzo M, Amato G, et al. Terminal ligature of inferior thyroid artery branches during total thyroidectomy for multinodular goiter is associated with higher postoperative calcium and PTH levels. J Visc Surg 2010;147:e329-32.

56. Smith MA, Jarosz H, Hessel P, et al. Parathyroid autotransplantation in total thyroidectomy. Am Surg 1990;56:404-6.

57. Shaha AR, Burnett C, Jaffe BM. Parathyroid autotransplantation during thyroid surgery. J Surg Oncol 1991;46:21-4.

58. Roh JL, Kim JM, Park CI. Central compartment reoperation for recurrent/persistent differentiated thyroid cancer: patterns of recurrence, morbidity, and prediction of postoperative hypocalcemia. Ann Surg Oncol 2011; 18:1312-8.

59. Zhao W, You L, Hou X, et al. The Effect of Prophylactic Central Neck Dissection on Locoregional Recurrence in Papillary Thyroid Cancer After Total Thyroidectomy: A Systematic Review and Meta-Analysis: pCND for the
Locoregional Recurrence of Papillary Thyroid Cancer. Ann Surg Oncol 2017;24:2189-98.

60. Roh JL, Yoon YH, Park CI. Chyle leakage in patients undergoing thyroidectomy plus central neck dissection for differentiated papillary thyroid carcinoma. Ann Surg Oncol 2008;15:2576-80.

61. Lee YS, Kim BW, Chang HS, et al. Factors predisposing to chyle leakage following thyroid cancer surgery without lateral neck dissection. Head Neck 2013;35:1149-52.

62. Rodgers GK, Johnson JT, Petruzzelli GJ, et al. Lipid and volume analysis of neck drainage in patients undergoing neck dissection. Am J Otolaryngol 1992;13:306-9.

63. Crumley RL, Smith JD. Postoperative chylous fistula prevention and management. Laryngoscope 1976;86:804-13.

64. Kim MK, Mandel SH, Baloch Z, et al. Morbidity following central compartment reoperation for recurrent or persistent thyroid cancer. Arch Otolaryngol Head Neck Surg 2004;130:1214-6.

65. Cayonu M, Acar A, Eryilmaz A, et al. Surgical approach and outcomes for revision surgery of the central neck compartment. J Craniofac Surg 2014;25:1797-800.

66. American Thyroid Association (ATA) Guidelines Taskforce on Thyroid Nodules and Differentiated Thyroid Cancer, Cooper DS, Doherty GM, et al. Revised American Thyroid Association management guidelines for patients with thyroid nodules and differentiated thyroid cancer. Thyroid 2009;19:1167-214.

67. Roh JL, Park JY, Kim JM, et al. Use of preoperative ultrasonography as guidance for neck dissection in patients with papillary thyroid carcinoma. J Surg Oncol 2009;99:28-31.

68. Levin KE, Clark AH, Duh QY, et al. Reoperative thyroid surgery. Surgery 1992;111:604-9.

69. Goretzki PE, Simon D, Frilling A, et al. Surgical reintervention for differentiated thyroid cancer. Br J Surg 1993;80:1009-12.

70. Pattou F, Combemale F, Fabre S, et al. Hypocalcemia following thyroid surgery: incidence and prediction of outcome. World J Surg 1998;22:718-24.

71. Wingert DJ, Friesen SR, Iliopoulos JI, et al. Postthyroidectomy hypocalcemia. Incidence and risk factors. Am J Surg 1986;152:606-10.

72. Chao TC, Jeng LB, Lin JD, et al. Reoperative thyroid surgery. World J Surg 1997;21:644-7.

73. Shaha AR, Jaffe BM. Parathyroid preservation during thyroid surgery. Am J Otolaryngol 1998;19:113-7.

74. Onkendi EO, McKenzie TJ, Richards ML, et al. 
Reoperative experience with papillary thyroid cancer.

World J Surg 2014;38:645-52.

75. Sosa JA, Bowman HM, Tielsch JM, et al. The importance of surgeon experience for clinical and economic outcomes from thyroidectomy. Ann Surg 1998;228:320-30.

Cite this article as: Lombardi D, Accorona R, Paderno A, Cappelli C, Nicolai P. Morbidity of central neck dissection for papillary thyroid cancer. Gland Surg 2017;6(5):492-500. doi: 10.21037 /gs.2017.05.07
76. Adam MA, Thomas S, Youngwirth L, et al. Is There a Minimum Number of Thyroidectomies a Surgeon Should Perform to Optimize Patient Outcomes? Ann Surg 2017;265:402-7. 